\title{
Anaerobic Intrinsic Bioremediation of Benzene in the Presence of Salinity
}

\author{
Kelvin K. Chan, Ania C. Urich \\ University of Alberta, Department of Civil and Environmental Engineering \\ 9211 - 116 Street NW, Edmonton, Alberta, Canada \\ kkchan2@ualberta.ca; aurich@ualebrta.ca
}

\section{Extended Abstract}

Due to dependence on petroleum hydrocarbons (PHC) as a fuel source, soils and groundwaters containing PHC are one of the most frequent forms of contamination in Canada and presents as a multibillion dollar problem due to the numerous and diverse conditions of contaminated sites [1]. Within the countless chemicals classified as PHCs, benzene is a distinguished environmental contaminant due to its recalcitrant, mobile, and toxic nature. Releases of benzene are most commonly associated to underground storage tank leakages, inadvertent spills, and inappropriate waste disposal methods within the petroleum industry [2]. In Canada alone, 34, 1, and 0.2 kilotons of benzene are estimated to be annually released into the atmosphere, water, and soil respectively [3].

In contrast to traditional physical and chemical remediation methods, intrinsic bioremediation is an alternative strategy utilizing indigenous microbial communities within a contaminated site to degrade benzene. When applicable, bioremediation is an attractive option due to its minimal operating costs, lack of on-site operation, and non-intrusive nature. While aerobic bioremediation is a topic well researched and a remediation method commonly used, sites often become oxygen deficient or anaerobic due to the high oxygen demand imposed by the benzene load [4]. Upon oxygen depletion, microorganisms must rely on the next readily available electron acceptor to metabolize benzene. In most cases, such electron acceptors exists in the form of nitrate, manganese (IV), iron (III), sulfate, and carbon dioxide [5]. This transition between aerobic and anaerobic biodegradation often tends to be the stalling point in many contaminated sites due to the absence of a microbial culture capable of utilizing the previously mentioned electron acceptors in anaerobic conditions or simply an insufficient amount of these electron acceptors [6].

In addition to PHC contamination, many upstream drilling sites and downstream processing facilities within the petroleum industry are plagued by elevated salt content due to incidental releases of hypersaline produced waters which simultaneously contain both benzene and salt [6]. To date, no research has been identified which studies the biodegradation of benzene under both saline and anaerobic conditions.

This research seeks to explore the effect of salinity on the anaerobic biodegradation of benzene. Microcosms generated from pristine soil samples originating from underneath the area currently known as the Suncor South Tailings Pond in Fort McMurray, Alberta, were developed in 2011 and have since been conditioned to utilize benzene as the sole carbon source. Using these microcosms, biodegradation is observed under anaerobic nitrate-, sulfate- reducing, and methanogenic conditions in varying salt concentrations $(0.5,1.0$, and $2.0 \mathrm{~g} / \mathrm{L} \mathrm{NaCl})$. Benzene is monitored on a weekly basis via GC-FID (Gas Chromatography - Flame Ionization Detection) and sufficient electron acceptor content is ensured via IC (Ion Chromatography). In the preliminary study, marginally greater biodegradation rates were observed at the $0.5 \mathrm{~g} / \mathrm{L} \mathrm{NaCl}$ salinity level for nitrate-reducing and methanogenic conditions while sulfate-reducing conditions favored $2.0 \mathrm{~g} / \mathrm{L} \mathrm{NaCl}$. Following a lag phase, exponential benzene degradations rates were $0.1645,0.2412$, and $0.2486 \mathrm{mg} \cdot \mathrm{L}^{-1} \cdot \mathrm{d}^{-1}$ for nitrate-, sulfate-reducing, and methanogenic conditions respectively in the previous mentioned salinity levels. Furthermore, biomass samples were collected at the initiation of the study with the express interest of genomic analysis and comparison against a later time to observe shifts in the microbial community under varying salinity pressures.

Canada's petroleum industry, although controversial at times, continues to be a major international driving force in the fuel industry as well as an economic foundation within the country. With no foreseeable end of this dependence in sight, responsible and innovative management of PHC contaminated sites must be a cornerstone in the management framework within the petroleum industry. As salinity and benzene are often prevailing co-contaminants, this research aims to further the 
currently limited understanding of benzene anaerobic degradation under saline presence with the vision of lowering the environmental impact of the petroleum industry.

\section{References}

[1] Canadian Council Ministers of the Environment, Canada-wide standards for petroleum hydrocarbons (PHC) in soil. Ccme., pp. 1-8, 2014.

[2] S. A. Mancini, G. Lacrampe-Couloume, B. S. Lollar, "Source differentiation for benzene and chlorobenzene groundwater contamination: A field application of stable carbon and hydrogen isotope analyses," Environ. Forensics., vol. , pp. 177-186, 2008. doi:10.1080/15275920802119086.

[3] Government of Canada (GOC), Canadian Environmental Protection Act, 1999.

[4] H. G. Song, X. Wang, R. Bartha, "Bioremediation potential of terrestrial fuel spills," Appl. Environ. Microbiol., vol. 56, pp. 652-656, 1990.

[5] U. S. Environmental Protection Agency (EPA), Engineered approaches to in situ bioremediation of chlorinated solvents: Fundamentals and field applications, pp. 1-144, 2000. doi:EPA 542-R-00-008

[6] A. C. Ulrich, S. E. Guigard, J. M. Foght, K. M. Semple, K. Pooley, J. E. Armstrong, K.W. Biggar, "Effect of salt on aerobic biodegradation of petroleum hydrocarbons in contaminated groundwater," Biodegradation., vol. 20, pp. 2738, 2009. doi:10.1007/s10532-008-9196-0. 